\title{
Characterization of Chlorophenol 4-Monooxygenase (TftD) and NADH:Flavin Adenine Dinucleotide Oxidoreductase (TftC) of Burkholderia cepacia AC1100
}

\author{
Michelle R. Gisi† and Luying Xun* \\ School of Molecular Biosciences, Washington State University, Pullman, Washington
}

Received 23 December 2002/Accepted 17 February 2003

\begin{abstract}
Burkholderia cepacia AC1100 uses 2,4,5-trichlorophenoxyacetic acid, an environmental pollutant, as a sole carbon and energy source. Chlorophenol 4-monooxygenase is a key enzyme in the degradation of 2,4,5trichlorophenoxyacetic acid, and it was originally characterized as a two-component enzyme (TftC and TftD). Sequence analysis suggests that they are separate enzymes. The two proteins were separately produced in Escherichia coli, purified, and characterized. TftC was an NADH:flavin adenine dinucleotide (FAD) oxidoreductase. A C-terminally His-tagged fusion TftC used NADH to reduce either FAD or flavin mononucleotide (FMN) but did not use NADPH or riboflavin as a substrate. Kinetic and binding property analysis showed that FAD was a better substrate than FMN. TftD was a reduced FAD $\left(\mathrm{FADH}_{2}\right)$-utilizing monooxygenase, and FADH was supplied by TftC. It converted 2,4,5-trichlorophenol to 2,5-dichloro-p-quinol and then to 5-chlorohydroxyquinol but converted 2,4,6-trichlorophenol only to 2,6-dichloro-p-quinol as the final product. TftD interacted with $\mathrm{FADH}_{2}$ and retarded its rapid oxidation by $\mathrm{O}_{2}$. A spectrum of possible TftD-bound FAD-peroxide was identified, indicating that the peroxide is likely the active oxygen species attacking the aromatic substrates. The reclassification of the two enzymes further supports the new discovery of $\mathrm{FADH}_{2}$-utilizing enzymes, which have homologues in the domains Bacteria and Archaea.
\end{abstract}

2,4,5-Trichlorophenoxyacetic acid $(2,4,5-\mathrm{T})$ is an herbicide and a major component of Agent Orange (3, 11, 27). As a chlorinated pollutant, it is recalcitrant to degradation in the environment (11). Burkholderia cepacia AC1100 is the first bacterium in pure culture shown to use $2,4,5-\mathrm{T}$ as a sole carbon and energy source $(21,22)$. The $2,4,5$-T degradation pathway has been completely elucidated for AC1100 (44). TftAB, a 2,4,5-T oxygenase, converts 2,4,5-T to 2,4,5-trichlorophenol $(2,4,5-\mathrm{TCP})(5,43)$. TftCD catalyzes the oxidation of $2,4,5-$ TCP to 2,5-dichloro- $p$-quinol (also known as 2,5-dichloro- $p$ hydroquinone, 2,5-DiCH) and then to 5-chlorohydroxyquinol (5-CHQ) $(17,41)$. TftG dechlorinates 5-CHQ to hydroxyquinone, which is reduced to hydroxyquinol by a quinone reductase (44). TftH breaks the aromatic ring of hydroxyquinol to yield maleylacetate; TftE reduces maleylacetate to 3-oxoadipate, which is further channeled into the tricarboxylic acid cycle for complete mineralization (6).

$\mathrm{TftC}$ and TftD were originally characterized as a two-component chlorophenol 4-monooxygenase (17, 41). Recent sequence analysis suggests that TftD may belong to the newly discovered reduced flavin adenine dinucleotide $\left(\mathrm{FADH}_{2}\right)$-utilizing monooxygenases, which currently consist of only two characterized enzymes, 4-hydroxyphenylacetate 3-monooxygenase $(\mathrm{HpaB})$ of Escherichia coli $\mathrm{W}(13,42)$ and 2,4,6-trichlorophenol monooxygenase (TcpA) of Ralstonia eutropha JMP134 (28). Surprisingly, a new report stated that TftD alone, without

\footnotetext{
* Corresponding author. Mailing address: School of Molecular Biosciences, Abelson Hall 301, Washington State University, Pullman, WA 99164-4324. Phone: (509) 335-2787. Fax: (509) 335-1907. E-mail: xun@mail.wsu.edu.

$\dagger$ Present address: Battelle Dugway Operations, Special Programs Division, Dugway, UT 84022.
}

TftC, catalyzes NADH-dependent oxidation of chlorophenols and questioned whether TftD is an $\mathrm{FADH}_{2}$-utilizing monooxygenase (30). To support the assignment by sequence analysis and to clarify the discrepancy, we characterized TftC and TftD as two separate enzymes: an NADH:FAD oxidoreductase and an $\mathrm{FADH}_{2}$-utilizing monooxygenase.

\section{MATERIALS AND METHODS}

Bacterial strains and culture conditions. E. coli strains NovaBlue and BL21(DE3) were grown in Luria-Bertani (LB) medium or on LB agar (36) with kanamycin $(30 \mu \mathrm{g} / \mathrm{ml})$ at $37^{\circ} \mathrm{C}$. Strain BL21(DE3) was also incubated at room temperature when used to overproduce recombinant proteins.

Gene cloning and protein expression. PCR primers were designed to clone $t f t C$ into pET-30 LIC vector (Novagen, Madison, Wis.) with a C-terminal six-His tag. The forward primer (5'-GAA-CGA-AGG-AGG-TTC-ATA-TGC-ATG-CCG$3^{\prime}$ ) was from the beginning of the $t f t C$ gene starting at position 875 of the deposited sequence (GenBank accession no. U83405), and an NdeI site was introduced (underlined) by changing one base. The reverse primer (5'-GGGGAA-TTC-AGG-CTT-ATT-CCG-CGA-GCG-3'), complementary to sequences from base positions 1418 to 1444, had an EcoRI site introduced (underlined) by altering two bases, which also fused the $\mathrm{C}$ terminus of $\mathrm{tfC}$ into a six-His tag on the vector. The gene was amplified from B. cepacia AC1100 genomic DNA for $30 \mathrm{PCR}$ cycles of $94^{\circ} \mathrm{C}$ for $40 \mathrm{~s}, 55^{\circ} \mathrm{C}$ for $30 \mathrm{~s}$, and $72^{\circ} \mathrm{C}$ for $40 \mathrm{~s}$. The amplification yielded a 542-bp PCR product. The product was cut with $\mathrm{NdeI}$ and EcoRI and then ligated into a similarly digested pET-30 LIC vector. The ligation product was electroporated into E. coli strain NovaBlue, and the clones were recovered and sequenced for confirmation (36). The correct clone was then transformed into E. coli BL21(DE3) for protein production.

To clone $t f t D$, PCR primers were synthesized. The forward primer $\left(5^{\prime}\right.$-TATGGA-GAC-TGC-ATA-TGC-GCA-CTG-3') started at base position 1457 (GenBank accession no. U83405), and an NdeI site was introduced (underlined) by changing three bases. The reverse primer (5'-CGG-AAT-TCG-CTA-CAC-TCTTGG-TAA-3'), complementary to sequences from positions 3053 to 3076 , had three bases changed to introduce an EcoRI site (underlined). The gene was amplified by PCR as described for $t f t C$ amplification, and a $1.6-\mathrm{kb}$ product was obtained. It was cloned into pET-30 LIC vector as a nonfusion gene and transformed into $E$. coli as described for $t f t C$ cloning. 
Protein purification. Cells were grown in $\mathrm{LB}$ medium at $37^{\circ} \mathrm{C}$ to a turbidity of 0.5 at $600 \mathrm{~nm}$, induced with $1 \mathrm{mM}$ isopropyl- $\beta$-D-thiogalactopyranoside, and then incubated at room temperature for $3 \mathrm{~h}$. The cells were harvested by centrifugation and suspended in $20 \mathrm{mM}$ potassium phosphate (KPi) buffer ( $\mathrm{pH} \mathrm{7.0)}$ Freshly prepared phenylmethylsulfonyl fluoride in absolute ethanol was added to a final concentration of $0.5 \mathrm{mM}$. The cells were disrupted by passage through a French pressure cell (model FA-030; Aminco, Urbana, Ill.) three times at 260 $\mathrm{MPa}$. The lysate was ultracentrifuged at $50,000 \times g$ for $1 \mathrm{~h}$ to remove cell debris and membrane fragments. All purification steps were performed at $4^{\circ} \mathrm{C}$. Fre, a general flavin reductase from $E$. coli, was purified as previously reported (42).

(i) Purification of His-tagged $\mathbf{T f t C}\left(\mathbf{T f t C}_{\mathbf{H}}\right)$. Five hundred milliliters of cells was harvested and resuspended in $10 \mathrm{ml}$ of the KPi buffer. The cells were lysed. Four milliliters of the extract containing about $10 \mathrm{mg}$ of protein was shaken with $1 \mathrm{ml}$ of $\mathrm{Ni}^{2+}$-nitrilotriacetic acid (NTA) agarose beads (Qiagen, Valencia, Calif.) for $1 \mathrm{~h}$ at $4^{\circ} \mathrm{C}$ for binding. The resin was packed into a small column by gravity and was washed with $3 \mathrm{ml}$ of the wash solution. $\mathrm{TftC}_{\mathrm{H}}$ was then eluted off the column with $4 \mathrm{ml}$ of elution buffer. The wash solution contained $50 \mathrm{mM}$ sodium phosphate (pH 8.0), $300 \mathrm{mM} \mathrm{NaCl}, 1 \mathrm{mM}$ dithiothreitol (DTT), 10\% glycerol, and $40 \mathrm{mM}$ imidazole; the elution buffer contained all of the above with $140 \mathrm{mM}$ imidazole. The samples were stored at $-80^{\circ} \mathrm{C}$.

(ii) TftD purification. Cells from 2 liters of culture were harvested and suspended in $14 \mathrm{ml}$ of the KPi buffer. The cells were lysed, and the lysate was centrifuged. The supernatant was loaded onto a Cibicron Blue 3GA agarose column ( 1.5 by $18 \mathrm{~cm}$; Sigma, St. Louis, Mo.) equilibrated with the KPi buffer (pH 7) containing $1 \mathrm{mM}$ DTT. The unbound proteins were washed with 3 bed volumes of the KPi buffer, and TftD was eluted with 3 bed volumes of the KPi buffer containing $1 \mathrm{mM}$ DTT and $1 \mathrm{M} \mathrm{NaCl}$. The sample was concentrated to about $2 \mathrm{ml}$ by dialysis against dry Aquacide II (Calbiochem, La Jolla, Calif.) and dialyzed for $2 \mathrm{~h}$ against the KPi buffer containing $1 \mathrm{mM}$ DTT. The dialyzed sample was loaded onto a 2-ml Bio-Scale ceramic hydroxyapatite 2-I (CHT) column (Bio-Rad Laboratories, Hercules, Calif.) equilibrated with the KP buffer. TftD did not bind to the column and came off in a 4-ml wash with the equilibrating buffer. The sample was stored at $-80^{\circ} \mathrm{C}$.

Enzyme assays. NADH:FAD oxidoreductase activity was determined spectrophotometrically by measuring NADH oxidation $\left(\varepsilon_{340}=6,220 \mathrm{M}^{-1} \mathrm{~cm}^{-1}\right)$ in 20 mM KPi buffer ( $\mathrm{pH} 7.0$ ) containing $20 \mu \mathrm{M}$ FAD and $300 \mu \mathrm{M}$ NADH at room temperature $\left(24^{\circ} \mathrm{C}\right)$. One unit of reductase activity was defined as the amount required to catalyze the oxidation of $1 \mathrm{nmol}$ of NADH per min.

Chlorophenol 4-monooxygenase activity was measured by analyzing the conversion of 2,4,6-TCP to 2,6- $\mathrm{DiCH}$ with a high-performance liquid chromatography (HPLC) system. A 40- $\mu$ l assay mixture contained $20 \mathrm{mM}$ KPi buffer (pH 7.0), $10 \mu$ M FAD, $100 \mu \mathrm{M}$ 2,4,6-TCP, 0.4\% Tween 20, $1 \mathrm{mM}$ ascorbic acid, 20 $\mathrm{U}$ of TftC or Fre, $2.5 \mathrm{mM}$ NADH, and $10 \mathrm{U}$ ( $\mu \mathrm{mol} / \mathrm{min})$ of catalase (Sigma). The reaction was started by the addition of $\mathrm{NADH}$, the reaction mixture was incubated for $3 \mathrm{~min}$ at room temperature, and the reaction was terminated by adding $40 \mu \mathrm{l}$ of an acetonitrile-acetic acid (vol/vol, 9:1) mixture. The samples were centrifuged, and the supernatants were analyzed by HPLC. Stock solutions of $100 \mathrm{mM}$ 2,4,5-TCP, 2,4,6-TCP, 2,5-DiCH, and 2,6-DiCH were prepared in absolute ethanol.

Kinetic analysis was done by measuring the decrease of the substrate. Three independent sets of experiments were performed with at least six substrate concentrations ranging from one-half of $K_{m}$ to four times $K_{m}$. Data were fitted with the Michaelis-Menten equation, by using Grafit 5.0 (R. J. Leatherbarrow, Erithicus Software Ltd., Staines, England, 2001).

Measurement of the dissociation constant. The dissociation constants, $K_{d}$, of the $\mathrm{TftC} \cdot \mathrm{FAD}$ and $\mathrm{TftC} \cdot$ flavin mononucleotide (FMN) complexes were measured by using a fluorometer (Luminescence Spectrometer LS50B; PerkinElmer, Shelton, Conn.). The excitation wavelength was set at $280 \mathrm{~nm}$, and the fluorescence emission of $\mathrm{TftC}_{\mathrm{H}}$ was recorded at $350 \mathrm{~nm}$. The excitation and emission monochromator slit widths were set at $2.5 \mathrm{~nm}$. A 2-ml solution of $1 \mu \mathrm{M}$ $\mathrm{TftC}_{\mathrm{H}}$ in $50 \mathrm{mM} \mathrm{KPi}$ buffer ( $\mathrm{pH} 7.0$ ) was titrated with flavin from a $1 \mathrm{mM}$ stock solution, and the fluorescence was measured after each addition. The concentration of $\mathrm{TftC}_{\mathrm{H}}$ - flavin complex was estimated by the following equation:

$$
\left[\mathrm{TftC}_{\mathrm{H}} \cdot \text { flavin }\right]=\left[\mathrm{TftC}_{\mathrm{H}}\right] \times\left[\left(I_{0}-I_{c}\right) /\left(I_{0}-I_{f}\right)\right]
$$

In the equation, $\left[\mathrm{TftC}_{\mathrm{H}}\right]$ represents the initial concentration of $\mathrm{TftC}_{\mathrm{H}}, I_{0}$ is the fluorescence intensity of $\mathrm{TftC}_{\mathrm{H}}$ at the initial titration point, $I_{c}$ is the fluorescence intensity of $\mathrm{TftC}_{\mathrm{H}}$ at a specific titration point, and $I_{f}$ is the fluorescence intensity at saturating concentrations of flavin. The $K_{d}$ was determined from a plot of [ $\mathrm{TftC}_{\mathrm{H}} \cdot$ flavin] ( $y$ axis) versus [total flavin] ( $x$ axis) fitted with equation 2 (32), by using Grafit 5.0. Cap was the binding capacity of $\mathrm{TftC}_{\mathrm{H}}$.

$$
y=\frac{-\left(K_{d}+x+C a p\right)+\sqrt{\left(C a p+x+K_{d}\right)^{2}-4 x C a p}}{2}
$$

pH, ionic strength, and temperature optima. $\mathrm{TftC}_{\mathrm{H}}$ activity was measured at $\mathrm{pH}$ levels ranging from 5 to 6.2 in $40 \mathrm{mM}$ sodium succinate buffer and from 6 to 7.6 in $40 \mathrm{mM}$ sodium phosphate $(\mathrm{NaPi})$ buffer in a total volume of $1 \mathrm{ml}$ at $30^{\circ} \mathrm{C}$. The reaction mixture was the same as described for the enzyme assay. The enzyme activity at ionic strengths ranging from 10 to $160 \mathrm{mM} \mathrm{NaPi}(\mathrm{pH} 7.0)$ was tested in a similar way. The temperature optimum was determined within the range of 23 to $45^{\circ} \mathrm{C}$ in $20 \mathrm{mM} \mathrm{NaPi}(\mathrm{pH} 7.0)$.

Oxygen consumption. Oxygen consumption was measured in a closed reaction vessel (0.650-ml total volume) fitted with a Clark-type oxygen electrode (Instech, Plymouth Meeting, Pa.). The electrode was calibrated by using $N$-methylphenazonium methosulfate and NADH to quantitatively consume $\mathrm{O}_{2}(35)$. The reaction mixture contained $2.2 \mu \mathrm{g}$ of Fre alone or Fre with $6.8 \mu \mathrm{M} \mathrm{TftD}$. The reaction mixture also contained $2 \mu \mathrm{M}$ FAD, $100 \mu \mathrm{M}$ NADH, $1 \mathrm{mM}$ ascorbic acid, and $100 \mu \mathrm{M}$ 2,4,6-TCP. Catalase (99 U) was added after the oxygen consumption stopped. After the reaction was completed, the consumption of 2,4,6-TCP was measured by HPLC.

Analytical methods. A UV-visible light spectrophotometer (Ultrospec 4000; Amersham Pharmacia, Piscataway, N.J.) was used to analyze absorption spectral changes during FAD reduction. The data were recorded with a SWIFT program (Amersham Pharmacia) on a personal computer and transferred to Microsoft (Redmond, Wash.) Excel format. The influence of a compound on the spectrum of a mixture can be removed by subtracting the spectrum of the single compound from that of the mixture. The subtraction and generation of figures with digital data were done by using Microsoft Excel.

An HPLC system (Waters, Milford, Mass.) with a Biosep Sec-S3000 sizeexclusion column (7.8 by $300 \mathrm{~mm}$; Phenomenex, Torrance, Calif.) was used to determine the native molecular weights of the proteins. The column was equilibrated with $100 \mathrm{mM} \mathrm{KPi}(\mathrm{pH} 7.0)$ at a flow rate of $0.5 \mathrm{ml} / \mathrm{min}$. The standards (Bio-Rad) were blue dextran $(2,000,000 \mathrm{Da})$, bovine serum albumin $(66,000 \mathrm{Da})$, carbonic anhydrase $(29,000 \mathrm{Da})$, cytochrome $c(12,400 \mathrm{Da})$, and aprotinin $(6,500$ Da). The HPLC system with a Nova-Pak $\mathrm{C}_{18}$ column (3.9 by $150 \mathrm{~mm}$; Waters) was used to analyze 2,4,5-TCP, 2,4,6-TCP, 2,5-DiCH, and 2,6-DiCH. The compounds were eluted by an $11 \mathrm{mM} \mathrm{H}_{3} \mathrm{PO}_{4}$-acetonitrile gradient. The percentages of acetonitrile were as follows: 5 to $70 \%, 5$-ml linear gradient; $70 \%, 6 \mathrm{ml}$; and $100 \%, 3 \mathrm{ml}$. The absorption spectra from 240 to $350 \mathrm{~nm}$ were monitored by a Waters 996 photodiode array detector operated with the Millennium 2010 version 2.1 program (Waters) on a personal computer. The compounds were identified by comparing their retention times and absorption spectra to those of authentic standards, and the peak areas were used for quantification.

The end product of 2,4,5-TCP oxidation by TftD was determined by mass spectrometry (MS). An 0.6-ml reaction mixture contained $2 \mu \mathrm{M}$ FAD, $1 \mathrm{mM}$ ascorbic acid, $100 \mu \mathrm{M}$ 2,4,5-TCP, $2.5 \mathrm{mM}$ NADH, $60 \mathrm{U}$ of catalase, $2.2 \mu \mathrm{g}$ of Fre, $10 \%$ glycerol, and $4.25 \mu \mathrm{M} \mathrm{TftD}$ in $20 \mathrm{mM} \mathrm{KPi} \mathrm{(pH} \mathrm{7.0).} \mathrm{The} \mathrm{reaction} \mathrm{was}$ carried out at room temperature for $20 \mathrm{~min}$, and HPLC analysis confirmed the complete degradation of 2,4,5-TCP. The organic compounds were extracted into ethyl acetate, dried, derivatized with acetic anhydride, and analyzed on a QP5050A gas chromatography (GC)-MS system (Shimadzu, Columbia, Md.) as previously described (28).

Sodium dodecyl sulfate-polyacrylamide gel electrophoresis (SDS-PAGE) was carried out by the method of Laemmli (24), and gels were stained with GelCode Blue (Pierce, Rockford, Ill.). Protein concentrations were determined by using a protein dye reagent (2) with bovine serum albumin as the standard.

\section{RESULTS}

Protein production and purification. To determine whether $\mathrm{TftC}$ and TftD are separate enzymes, the $t f t C$ and $t f t D$ genes were cloned separately into pET-30 LIC vector (Novagen) and transformed into $E$. coli strain BL21(DE3) for overexpression. When induced at $37^{\circ} \mathrm{C}$, the proteins were mainly insoluble. However, the cells produced soluble $\mathrm{TftC}$ and $\mathrm{TftD}$ proteins when incubated at room temperature after induction.

$\mathrm{TftC}$ was produced as a C-terminally His-tagged fusion protein, $\mathrm{TftC}_{\mathrm{H}}$, and was purified with $\mathrm{Ni}^{2+}$-NTA agarose beads. When $2.7 \mathrm{~g}$ (wet weight) of cells was disrupted, $23.1 \mathrm{mg}$ of protein was obtained in the cell extract with a specific activity of 7,040 $\mathrm{nmol} \mathrm{\textrm {min } ^ { - 1 } \mathrm { mg } \text { of protein }}{ }^{-1}$. The $\mathrm{Ni}^{2+}$-NTA agarose 


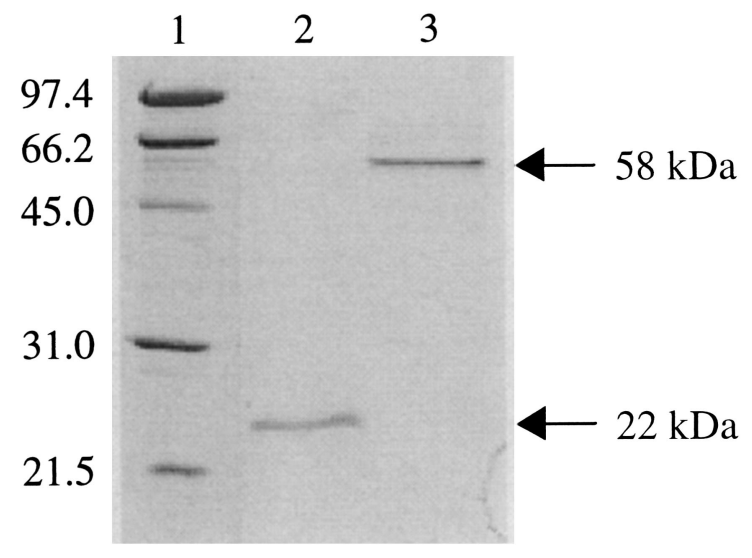

FIG. 1. SDS-PAGE of $\mathrm{TftC}_{\mathrm{H}}$ and TftD. Lane 1, molecular mass standards in kilodaltons (Bio-Rad); lane 2, $2.5 \mu \mathrm{g}$ of purified $\mathrm{TftC}_{\mathrm{H}}$; lane 3, $2 \mu \mathrm{g}$ of purified TftD.

purification was sufficient to purify $\mathrm{TftC}_{\mathrm{H}}$ to apparent homogeneity (Fig. 1). When $9.2 \mathrm{mg}$ of cell extract protein was applied to $1 \mathrm{ml}$ of $\mathrm{Ni}^{2+}$-NTA agarose beads, $0.17 \mathrm{mg}$ of $\mathrm{TftC}_{\mathrm{H}}$ was purified with a specific activity of $49,000 \mathrm{nmol} \mathrm{min}{ }^{-1} \mathrm{mg}$ of protein $^{-1}$. TftD was produced as a nonfusion protein and purified with Cibicron Blue 3GA resin and a prepacked hydroxyapatite column. From $7.8 \mathrm{~g}$ (wet weight) of cells, $57.3 \mathrm{mg}$ of protein was obtained in the cell extract. After purification, $4.0 \mathrm{mg}$ of $\mathrm{TftD}$ was purified to apparent homogeneity (Fig. 1). The specific activity of purified TftD was $280 \mathrm{nmol} \mathrm{min}{ }^{-1} \mathrm{mg}$ of protein ${ }^{-1}$ for 2,4,6-TCP oxidation. The TftD was shown to be $58 \mathrm{kDa}$ and $\mathrm{TftC}_{\mathrm{H}}$ was shown to be $22 \mathrm{kDa}$ by SDS-PAGE. Both $\mathrm{TftD}$ and $\mathrm{TftC}_{\mathrm{H}}$ were monomers as estimated by sizeexclusion chromatography analysis. Pure $\mathrm{TftC}_{\mathrm{H}}$ was unstable in the elution buffer containing $140 \mathrm{mM}$ imidazole on ice, losing about $50 \%$ activity in $2 \mathrm{~h}$. It was relatively stable when kept at $-80^{\circ} \mathrm{C}$, where it lost about $50 \%$ activity after 2 months. TftD was relatively stable on ice for several hours and did not show any apparent loss of activity after storage at $-80^{\circ} \mathrm{C}$ for 2 months. The purified $\mathrm{TftC}_{\mathrm{H}}$ and $\mathrm{TftD}$ were both colorless, indicating that they do not have bound flavin prosthetic groups.

Enzyme activity and substrate specificity. $\mathrm{TftC}_{\mathrm{H}}$ used NADH to reduce either FAD or FMN, but it did not use NADPH or riboflavin as a substrate. The kinetic parameters of $\mathrm{TftC}_{\mathrm{H}}$ were determined (Table 1), and FAD was a better

TABLE 1. Kinetic properties of $\operatorname{TftC}(\mathrm{H})^{a}$

\begin{tabular}{lrcc}
\hline \multicolumn{1}{c}{ Substrate } & $K_{m}(\mu \mathrm{M})$ & $k_{\text {cat }}\left(\mathrm{s}^{-1}\right)$ & $\begin{array}{c}k_{\text {cat }} / K_{m} \\
\left(\mu \mathrm{M}^{-1} \mathrm{~s}^{-1}\right)\end{array}$ \\
\hline $\mathrm{FAD}^{b}$ & $4.8 \pm 1.5$ & $16.6 \pm 1.3$ & 3.4 \\
$\mathrm{FMN}^{b}$ & $10.3 \pm 4.1$ & $18.5 \pm 1.9$ & 1.8 \\
$\mathrm{Riboflavin}^{c}$ & $40.1 \pm 12.9$ & $30.1 \pm 4.4$ & 0.8 \\
$\mathrm{NADH}_{\mathrm{FAD}}{ }^{d}$ & $164.0 \pm 31.2$ & $11.9 \pm 0.9$ & 0.1 \\
$\mathrm{NADH}_{\mathrm{FMN}}{ }^{e}$ & & & \\
$\mathrm{NADPH}^{c}$ & & & \\
\hline
\end{tabular}

\footnotetext{
${ }^{a}$ Experiments were done in $20 \mathrm{mM} \mathrm{KPi}$ buffer $(\mathrm{pH} 7.0)$ at $24^{\circ} \mathrm{C}$. Values are means of three experiments with standard deviations.

${ }^{b}$ Determined with fixed NADH concentration at $300 \mu \mathrm{M}$

${ }^{c}$ No detectable activities.

${ }^{d}$ Determined with fixed FAD concentration at $18 \mu \mathrm{M}$.

${ }^{e}$ Determined with fixed FMN concentration at $20 \mu \mathrm{M}$.
}

TABLE 2. Kinetic properties of $\mathrm{TftD}^{a}$

\begin{tabular}{lrcc}
\hline Substrate & $K_{m}(\mu \mathrm{M})$ & $k_{\text {cat }}\left(\mathrm{s}^{-1}\right)$ & $\begin{array}{c}k_{\text {cat }} / K_{m} \\
\left(\mu \mathrm{M}^{-1} \mathrm{~s}^{-1}\right)\end{array}$ \\
\hline 2,4,5-TCP & $35.8 \pm 3.9$ & $0.67 \pm 0.03$ & 0.019 \\
2,5-DiCH & $4.3 \pm 1.1$ & $0.10 \pm 0.01$ & 0.025 \\
2,4,6-TCP & $39.9 \pm 7.6$ & $0.41 \pm 0.03$ & 0.010 \\
2,6-DiCH & & &
\end{tabular}

${ }^{a}$ Experiments were done in $40 \mathrm{mM} \mathrm{KPi}$ buffer $(\mathrm{pH} 7.0)$ at $24^{\circ} \mathrm{C}$. Values are means of triplicate experiments with standard deviations. TftD was the limiting factor with $\mathrm{TftC}_{\mathrm{H}}$ in excess, and so the $k_{\text {cat }}$ values are calculated with the molecular weight of $\mathrm{TftD}$.

${ }^{b}$ No detectable activities.

substrate than FMN. The highest enzyme activity was observed at pH 6.0 in NaPi buffer with 87, 82, 65, and $61 \%$ of the activity retained at $\mathrm{pH} 6.4,6.8,7.2$, and 7.6, respectively. Sodium succinate buffer was used for the $\mathrm{pH}$ range of 5.0 to 6.2. Compared to the activity in the $\mathrm{pH} 6.0 \mathrm{NaPi}$ buffer, 52, 64, 81, and $75 \%$ of its activity was retained at $\mathrm{pH} 5.0,5.4,5.8$, and 6.2 , respectively. The optimal temperature was seen at $30^{\circ} \mathrm{C}$ with $85,92,82$, and $70 \%$ activity at $23,35,40$, and $45^{\circ} \mathrm{C}$, respectively. The optimal ionic strength ranged from 10 to $120 \mathrm{mM} \mathrm{NaPi}$ ( $\mathrm{pH} 7$ ), supporting similar activity, which was reduced by $28 \%$ at $160 \mathrm{mM} \mathrm{NaPi}$.

TftD required a flavin reductase to supply $\mathrm{FADH}_{2}$. TftD oxidized 2,4,5-TCP, 2,4,6-TCP, and 2,5-DiCH but not 2,6$\mathrm{DiCH}$. The kinetic properties of $\mathrm{TftD}$ were determined with TftD to supply $\mathrm{FADH}_{2}$ (Table 2). When 2,4,5-TCP was used as a substrate, the product $2,5-\mathrm{DiCH}$ was readily degraded to 5-CHQ, whereas 2,4,6-TCP was quantitatively converted to 2,6-DiCH. Thus, the TftD activity was usually determined by measuring the oxidation of 2,4,6-TCP to 2,6-DiCH. To determine whether TftD was active with any flavin reductase or only with $\mathrm{TftC}$, Fre was used to replace $\mathrm{TftC}_{\mathrm{H}}$. The rates of 2,6$\mathrm{DiCH}$ production were similar when $\mathrm{TftD}$ was coupled with either $\mathrm{TftC}_{\mathrm{H}}$ or Fre in a typical $40-\mu$ l reaction mixture containing 10 to $100 \mathrm{U}$ of the flavin reductase.

Dissociation constants. The dissociation constant, $K_{d}$, was determined by measuring the fluorescent quenching of $\mathrm{TftC}_{\mathrm{H}}$ when FAD or FMN was added to the solution. Grafit was used to plot the added concentration of flavin versus bound flavin to obtain the $K_{d}$ values. The $K_{d}$ values were $2.2 \pm 0.1$ and $7.8 \pm$ $0.2 \mu \mathrm{M}$ for TftC-FAD and TftC-FMN complexes, respectively. The $K_{d}$ values correlated well with the $K_{m}$ values for FAD and FMN (Table 1).

End product analysis. HPLC analysis was used to show the complete consumption of 2,4,5-TCP by TftD and the formation of the end product. 2,4,5-TCP gave a peak at $9.0 \mathrm{~min}$, and the end product had a retention time of 5.08 min with an absorption maximum at $293 \mathrm{~nm}$; thus, it is proposed to be 5-CHQ because of its retention time and absorption maximum (41). The peak at $6.9 \mathrm{~min}$ for $2,5-\mathrm{DiCH}$ was transitorily observed during the reaction and was not detectable at the end of the reaction. The organic compounds were extracted into ethyl acetate, and the aqueous solution was analyzed with HPLC to confirm that the end product was completely extracted. The organic phase was dried and acetylated for GC-MS analysis. Three peaks were detected, with retention times of 8.72, 10.09, and $11.03 \mathrm{~min}$. The first peak had a mass spectrum typical of 
triacetylated glycerol, and the second peak was identified as monoacetylated ascorbate. Glycerol and ascorbate were reagents in the reaction mixture. The third peak had a mass spectrum that was typical for a molecule with one chlorine and was similar to the spectrum of triacetylated 6-CHQ as previously reported (28). A molecular ion peak was seen at $\mathrm{m} / \mathrm{z} 286$, and its fragments were at $\mathrm{m} / \mathrm{z} 244$ (loss of $-\mathrm{COCH}_{3}$ ), 202 (loss of two $-\mathrm{COCH}_{3}$ ), and 160 (loss of all three $-\mathrm{COCH}_{3}$ ). Since triacetylated 6-CHQ gave a different retention time of 10.65 min by the GC-MS analysis, the compound was assigned as triacetylated 5-CHQ.

The stoichiometry of TCP oxidation. The reaction stoichiometry of TCP oxidation by TftD coupled with Fre was analyzed by measuring NADH consumption, oxygen consumption, $\mathrm{H}_{2} \mathrm{O}_{2}$ production, and 2,4,6-TCP degradation. For $100 \mu \mathrm{M}$ NADH consumed, $66 \mu \mathrm{M} \mathrm{O}_{2}$ was consumed. A $34 \mu \mathrm{M}$ concentration of $\mathrm{H}_{2} \mathrm{O}_{2}$ was produced because $17 \mu \mathrm{M} \mathrm{O} \mathrm{O}_{2}$ was released upon addition of catalase. Since one $\mathrm{O}_{2}$ reacted with one $\mathrm{FADH}_{2}$ to generate one $\mathrm{H}_{2} \mathrm{O}_{2}$, this left $32 \mu \mathrm{M} \mathrm{O}_{2}$ for TCP oxidation. A $31 \mu \mathrm{M}$ concentration of TCP was consumed in the reaction, giving a ratio of $1: 1$ for oxygen consumption and TCP oxidation. The ratio of NADH used for TCP oxidation was approximately 2:1.

Spectral analysis of the effect of TftD on Fre activity. TftD required $\mathrm{FADH}_{2}$ as a cosubstrate, which was supplied by a flavin reductase. The flavin reductase reduces FAD with $\mathrm{NADH}$. In this study, the changes of NADH and FAD were monitored during the course of a reaction by scanning the absorption from 300 to $550 \mathrm{~nm}$. Fre was used as the flavin reductase in this experiment because it was more stable than $\mathrm{TftC}_{\mathrm{H}}$. The NADH consumption rate was higher in a reaction with Fre alone than in the same reaction also containing TftD (Fig. 2). When the reaction mixture contained only Fre, $\mathrm{FADH}_{2}$ was rapidly oxidized back to FAD without any apparent decrease of FAD $(10 \mu \mathrm{M})$ (Fig. 2B). In the presence of TftD (Fig. 2C), the FAD concentration was $1.95 \mu \mathrm{M}$ when estimated from the remaining absorption at $450 \mathrm{~nm}$ with the subtraction of the $\mathrm{FADH}_{2}$ absorption or it was $1.6 \mu \mathrm{M}$ when calculated by the Michaelis-Menten equation with the rate of NADH oxidation and the determined $K_{m}(1.4 \mu \mathrm{M})$ and $V_{\max }$ $(69,764 \mathrm{nmol} / \mathrm{min} / \mathrm{mg})$ for Fre under the assay condition.

Fre activity was analyzed by measuring the rate of NADH oxidation with various amounts of $\mathrm{TftD}$ in the reaction mixture. When TftD was absent in the reaction mixture, Fre activity was the highest (Fig. 3). As TftD concentration increased, the activity of Fre decreased, due to decreased FAD available in the reaction mixture. By the Michaelis-Menten equation, the FAD concentration in the reaction was estimated based on the rate of NADH oxidation and the kinetic parameters of Fre. The estimated available FAD concentration decreased from 1.65 to $0.10 \mu \mathrm{M}$ FAD when the TftD concentration was increased to $5 \mu \mathrm{M}$ (Fig. 3). Thus, TftD decreased FAD concentration during its reduction by Fre (Fig. 2 and 3 ), resulting in lower rates of NADH oxidation (Fig. 3).

A potential flavin-peroxide intermediate. The absorption spectrum of a reaction with TftD and Fre was analyzed to identify whether FAD-peroxide was present. The absorption spectrum of a reaction was monitored at different time points during the reaction. In the reaction, one $\mathrm{H}_{2} \mathrm{O}_{2}$ was produced from one $\mathrm{FADH}_{2}$ oxidation. When catalase was added, two
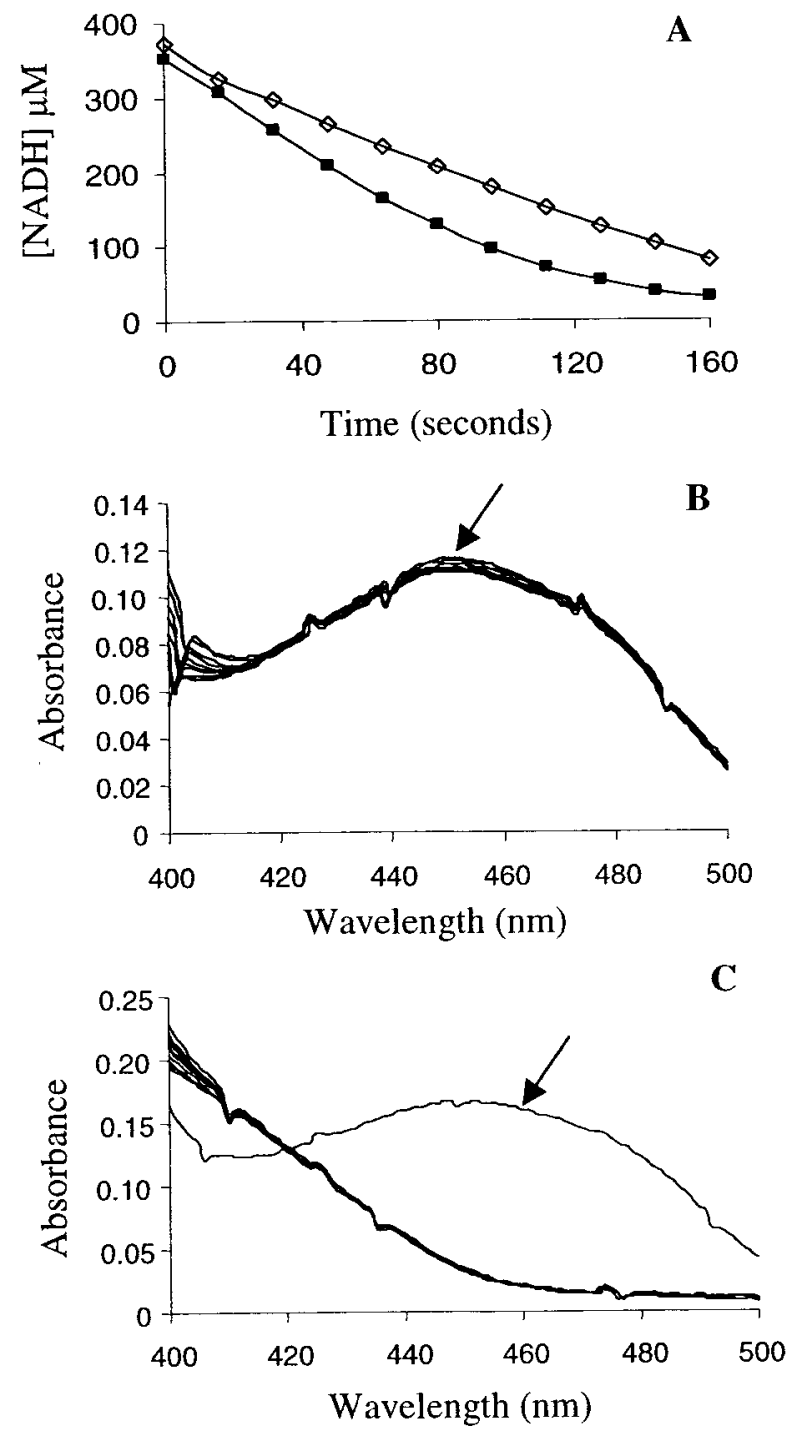

FIG. 2. UV-visible light spectra of NADH oxidation and FAD reduction by Fre alone and by Fre with TftD. The reaction mixture contained $2.2 \mu \mathrm{g}$ of Fre, $400 \mu \mathrm{M}$ NADH, $10 \mu \mathrm{M}$ FAD, and $99 \mathrm{U}$ of catalase in $0.7 \mathrm{ml}$ of $20 \mathrm{mM}$ KPi buffer ( $\mathrm{pH} 7.0$ ). In reaction mixtures containing TftD, $16.9 \mu \mathrm{M}$ TftD was used. (A) Time course of NADH oxidation in a reaction with Fre alone $(\boldsymbol{\square})$ or Fre with $\operatorname{TftD}(\diamond)$. (B) The FAD spectrum of the reaction with Fre alone. (C) The FAD spectrum of Fre and TftD. Arrows indicate the spectrum at time zero before the addition of Fre.

$\mathrm{H}_{2} \mathrm{O}_{2}$ were converted immediately back to one $\mathrm{O}_{2}$. Therefore, the reaction with catalase should theoretically consume approximately $510 \mu \mathrm{M} \mathrm{NADH}$, while the reaction without catalase should consume about half of that, assuming that $\mathrm{O}_{2}$ concentration is about $255 \mu \mathrm{M}$ (41). When the reaction without catalase completely depleted $\mathrm{O}_{2}$, NADH consumption stopped at about $125 \mu \mathrm{M}$ NADH (Fig. 4, line 2). At the same $\mathrm{NADH}$ concentration, the reaction with catalase at $130 \mathrm{~s}$ of $\mathrm{NADH}$ oxidation still had a sufficient amount of $\mathrm{O}_{2}$ due to regeneration by catalase, and $\mathrm{NADH}$ consumption continued (Fig. 2A and $\mathrm{C}$ and Fig. 4, line 1). Fig. 4, line 4 was obtained by subtracting the spectrum of $\mathrm{FADH}_{2}$ (Fig. 4, line 2) from the 


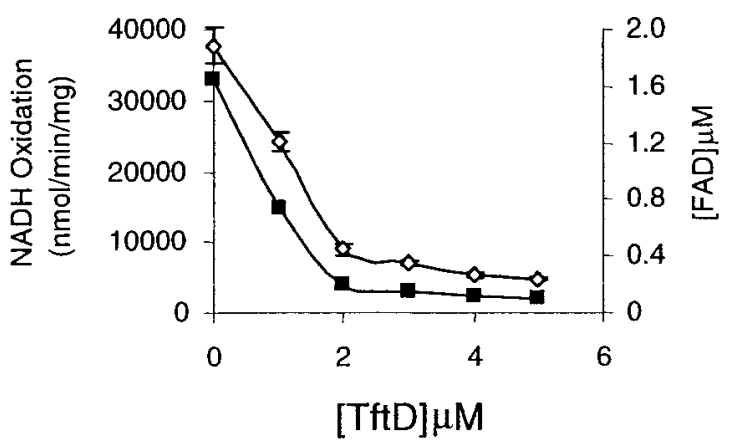

FIG. 3. The effect of TftD on NADH oxidation by Fre. The reaction mixture was similar to that described in the Fig. 2 legend except that the initial FAD concentration was $2 \mu \mathrm{M}$ and TftD was used from 0 to $5 \mu \mathrm{M}$. The rate of NADH oxidation $(\diamond)$ was determined by monitoring the decrease of NADH at $340 \mathrm{~nm}$. The FAD concentrations ( $\mathbf{\square})$ were estimated with the Michaelis-Menten equation by using the rates of $\mathrm{NADH}$ oxidation and the determined kinetic parameters of Fre.

spectrum of an FAD derivative (Fig. 4, line 1) in the presence of $\mathrm{O}_{2}$. The result was a peak with an absorbance maximum from 392 to $400 \mathrm{~nm}$, which was masked by the absorption of NADH in line 1. When NADH was completely depleted, FAD was regenerated due to complete oxidation (Fig. 4, line 3).

\section{DISCUSSION}

$\mathrm{TftC}$ and $\mathrm{TftD}$ were originally characterized as a two-component flavin-dependent monooxygenase (41). Our new data reclassify them as two individual enzymes: TftC is an NADH: $\mathrm{FAD}$ oxidoreductase, and $\mathrm{TftD}$ is an $\mathrm{FADH}_{2}$-utilizing monooxygenase. $\mathrm{NAD}(\mathrm{P}) \mathrm{H}$ :flavin oxidoreductases are divided into two classes: the class I enzymes do not have bound flavin prosthetic groups, and the class II enzymes do $(4,10,12,23$, 39). Since $\mathrm{TftC}_{\mathrm{H}}$ did not have bound flavin as a prosthetic group, it belongs to class I. The $\mathrm{TftC}_{\mathrm{H}}$ had a calculated mo-

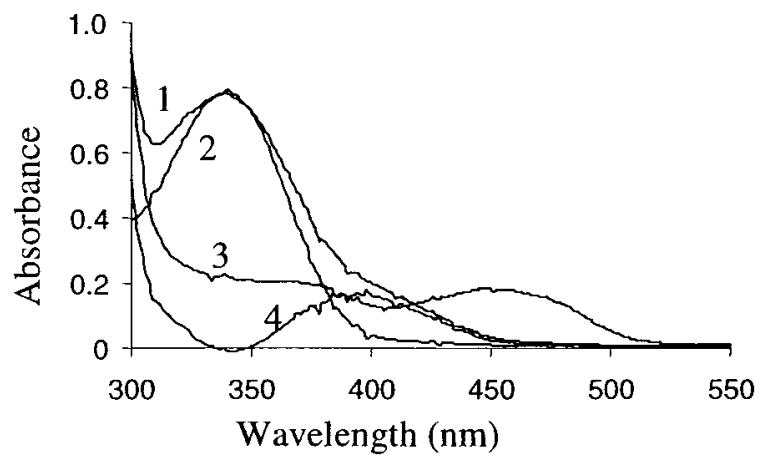

FIG. 4. Spectral analysis of a potential flavin intermediate during the reaction in the presence of TftD. Reaction conditions were essentially the same as described in the Fig. 2 legend with $16.9 \mu \mathrm{M}$ TftD in the presence or absence of $99 \mathrm{U}$ of catalase. Line 1, the spectrum of a reaction with catalase in the mixture was taken at $130 \mathrm{~s}$ of NADH oxidation; line 2 , the spectrum of a reaction without catalase was recorded when $\mathrm{O}_{2}$ was completely depleted at $200 \mathrm{~s}$ of the reaction; line 3 , the spectrum of the reaction with catalase was shown after $\mathrm{NADH}$ was used up and the FAD was regenerated at $350 \mathrm{~s}$ of the reaction; line 4 , the spectrum obtained by subtracting line 1 from line 2 .

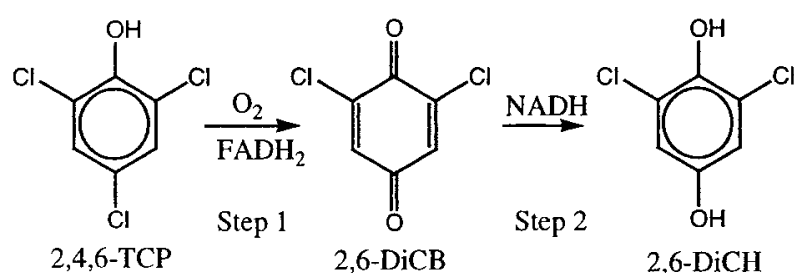

FIG. 5. Proposed conversion of 2,4,6-TCP to 2,6-DiCH. The first step is catalyzed by $\mathrm{TftD}$, and the second step is a chemical reduction by either $\mathrm{NADH}$ or $\mathrm{FADH}_{2} \cdot \mathrm{FADH}_{2}$ is produced by $\mathrm{TftC}_{\mathrm{H}}$ at the expense of NADH.

lecular weight of 21,194, which agrees with the SDS-PAGE analysis (Fig. 1). $\mathrm{TftC}_{\mathrm{H}}$ was active for $\mathrm{FAD}$ reduction with NADH as the reductant, and it did not use NADPH or riboflavin. Kinetic (Table 1) and binding properties show that $\mathrm{TftC}_{\mathrm{H}}$ prefers FAD to FMN. Its catalytic properties are different from those of other characterized flavin reductases. Although supplying $\mathrm{FADH}_{2}$ to $\mathrm{HpaB}$, which uses $\mathrm{FADH}_{2}$ to oxidize 4-hydroxyphenylacetate, $\mathrm{HpaC}$ reduces FMN faster than it reduces FAD (13). Fre, an E. coli general flavin reductase, can reduce riboflavin, FMN, and FAD with either NADH or NADPH (10). However, Fre reduces FMN faster than it does FAD when assayed individually, whereas FAD is the preferred substrate when both FAD and FMN are present due to the high affinity of Fre for FAD (29). Streptomyces viridifaciens has an NADPH:FAD oxidoreductase that reduces both FAD and FMN with either NADPH or NADH, with the highest rate being obtained for FAD reduction with NADPH as the reductant (33). Several flavin reductases have also been characterized from Vibrio sp., and they all prefer to reduce FMN $(19,26,45)$. Thus, to our knowledge, $\mathrm{TftC}_{\mathrm{H}}$ is the only characterized NADH:FAD oxidoreductase.

TftD has a calculated molecular weight of 57,451 , and it was shown to be a monomer by size-exclusion chromatography analysis. Compared to other $\mathrm{FADH}_{2}$-utilizing monooxygenases, TftD is similar in size and conserved in sequence. TcpA of $R$. eutropha JMP134 is a monomer of $60 \mathrm{kDa}$ (28), and $\mathrm{HpaB}$ of E. coli W is a homodimer with subunits of $59 \mathrm{kDa}$ (34). TftD had a broad substrate range and required oxygen and $\mathrm{FADH}_{2}$ to function. $\mathrm{FADH}_{2}$ was supplied by flavin reductases. The fact that Fre successfully replaced $\mathrm{TftC}_{\mathrm{H}}$ to provide TftD with $\mathrm{FADH}_{2}$ supports the idea that TftD is an $\mathrm{FADH}_{2}$ utilizing monooxygenase. Fre has also been used to supply $\mathrm{FADH}_{2}$ for TcpA (28) and HpaB (34). The kinetic properties of TftD were determined, and the parameters for 2,4,5- and 2,4,6-TCP are similar (Table 2). However, TftD degraded 2,5$\mathrm{DiCH}$ but not 2,6-DiCH, the direct products from the oxidation of 2,4,5-TCP and 2,4,6-TCP, respectively. In contrast to TftD, TcpA of $R$. eutropha JMP134 degrades 2,4,6-TCP to 6-CHQ with 2,6-DiCH as a possible intermediate (28). The stoichiometric analysis of 2,4,6-TCP oxidation by TftD and Fre shows the consumption of one $\mathrm{O}_{2}$ and two NADH for one 2,4,6-TCP oxidized to $2,6-\mathrm{DiCH}$. Thus, we propose that the direct product of 2,4,6-TCP oxidation by TftD is 2,6-dichloro$p$-quinone (2,6-DiCB), which is chemically reduced to 2,6DiCH by either NADH or $\mathrm{FADH}_{2}$ (Fig. 5). The formation of quinone after removal of a chlorine or a nitro group from substituted phenols by monooxygenases has been reported 
elsewhere $(15,18)$. We further speculate that TftD oxidizes 2,4,5-TCP to 2,5-DiCH and then converts 2,5-DiCH to 5-CHQ with reactions similar to those shown in Fig. 5, i.e., quinones are the direct products following each dechlorination step.

The end product of 2,4,5-TCP oxidation by TftD was shown to be 5-CHQ by GC-MS analysis. The mass spectrum of triacetylated $5-\mathrm{CHQ}$ is very similar to that of triacetylated 6-CHQ as previously reported (28) under the same GC-MS conditions. The different retention times of triacetylated 5-CHQ (11.03 min) and triacetylated 6-CHQ (10.65 min) are likely due to the difference in chlorine positions. The intermediate, 2,5-DiCH, was only transitorily accumulated and was completely converted to 5-CHQ when the reaction was complete. The observation is consistent with our kinetic data showing that 2,5-DiCH is a better substrate for TftD (Table 2) than is 2,4,5-TCP, so that it is not accumulated when 2,4,5-TCP is used up. Although 5-CHQ was previously reported as the end product based on HPLC analysis (41), a recent report stated that 5-CHQ was not detectable by HPLC analysis (30). The report also stated that TftD purified from B. cepacia AC1100 alone oxidizes chlorophenols. However, the reported SDSPAGE results clearly indicate that TftD is not purified to homogeneity, and the reported specific activity is much lower than that originally reported (41) or reported in this study. The data reported here confirm the original characterization of TftD and TftC for the oxidation of 2,4,5-TCP to 5-CHQ. The assignment of 5-CHQ as the end product of 2,4,5-TCP degradation by TftD is also consistent with the proposed pathway for 2,4,5-T degradation, as enzymes converting 5-CHQ into tricarboxylic acid cycle metabolic intermediates have been identified in B. cepacia AC1100 (44).

The analysis of the absorption spectra in a reaction with TftD and Fre reveals the presence of a potential FAD-peroxide (Fig. 4). The absorbance maximum of the possible FADperoxide was from 392 to $400 \mathrm{~nm}$, which is consistent with the absorption spectra of other flavin-peroxides (9). Equation 3 shows the two-step oxidation of $\mathrm{FADH}_{2}$ by $\mathrm{O}_{2}$.

$$
\mathrm{FADH}_{2}+\mathrm{O}_{2} \stackrel{k_{1}}{\longrightarrow} \text { FADHOOH } \stackrel{k_{2}}{\longrightarrow} \mathrm{FAD}+\mathrm{H}_{2} \mathrm{O}_{2}
$$

In this equation, $\mathrm{FADH}_{2}$ and $\mathrm{O}_{2}$ form $\mathrm{FAD}$-peroxide. In the absence of TftD, $k_{2}$ is faster than $k_{1}(31)$, and FAD-peroxide is not accumulated. Thus, the observed FAD-peroxide in the presence of TftD must be bound to TftD, and TftD slows down $k_{2}$ so that FAD-peroxide is accumulated and detectable by the spectrophotometer. The accumulation of FAD-peroxide is also consistent with the observed decrease of FAD (Fig. 2 and 3), which reduces Fre's activities by lowering the concentration of its substrate FAD in the reaction mixture. The binding and protection of FMN-peroxide by a bacterial luciferase, an $\mathrm{FMNH}_{2}$-utilizing monooxygenase, have been well documented; the enzyme uses the FMN-peroxide to attack its aldehyde substrate (16). Thus, it is likely that TftD attacks its aromatic substrate with the bound FAD-peroxide.

$\mathrm{FADH}_{2}$-utilizing monooxygenases are important enzymes in bioremediation for the degradation of aromatic compounds. Although there are only three enzymes characterized in this group to date, several proteins are candidates for being clas- sified as $\mathrm{FADH}_{2}$-utilizing monooxygenases because of sequence similarities $(1,7,8,14,20,37,38,40)$. The characterization of these proteins as $\mathrm{FADH}_{2}$-utilizing monooxygenases will help us to understand further the genetics and biochemistry of this unique enzyme family and to advance their application to bioremediation of pollutants or biosynthesis of specialty chemicals (25).

\section{ACKNOWLEDGMENTS}

We thank Tai-Man Louie, Linda Thomashow, and Lisa Gloss for their interest and helpful comments on this work.

Michelle Gisi was primarily supported by the school as a teaching assistant. This research was funded in part by NSF grant MCB9722970.

\section{REFERENCES}

1. Becker, D., T. Schrader, and J. Andreesen. 1997. Two-component flavin dependent pyrrole 2-carboxylate monooxygenase from Rhodococcus sp. Eur. J. Biochem. 249:739-747.

2. Bradford, M. M. 1976. A rapid and sensitive method for the quantitation of microgram quantities of protein utilizing the principle of protein-dye binding. Anal. Biochem. 72:248-254.

3. Chaudhry, G. O., and S. Chapalamadugu. 1991. Biodegradation of halogenated organic compounds. Microbiol. Rev. 55:59-79.

4. Coves, J., V. Niviere, M. Eschenbrenner, and M. Fontecave. 1993. NADPHsulfite reductase from Escherichia coli. A flavin reductase participating in the generation of the free radical of ribonucleotide reductase. J. Biol. Chem. 268: $18604-18609$.

5. Danganan, C. E., R. W. Ye, D. L. Daubaras, L. Xun, and A. M. Chakrabarty. 1994. Nucleotide sequence and functional analysis of the genes encoding 2,4,5-trichlorophenoxyacetic acid oxygenase in Pseudomonas cepacia AC1100. Appl. Environ. Microbiol. 60:4100-4106.

6. Daubaras, D. L. K. Saido, and A. M. Chakrabarty. 1996. Purification of hydroxyquinol 1,2-dioxygenase and maleylacetate reductase: the lower pathway of 2,4,5-trichlorophenoxyacetic acid metabolism by Burkholderia cepacia AC1100. Appl. Environ. Microbiol. 62:4276-4279.

7. Delaney, S. M., D. V. Mavrodi, R. F. Bonsall, and L. S. Thomashow. 2001. phzO, a gene for biosynthesis of 2-hydroxylated phenazine compounds in Pseudomonas aureofaciens 30-84. J. Bacteriol. 183:318-327.

8. Duffner, F. M., and R. Müller. 1998. A novel phenol hydroxylase and catechol 2,3-dioxygenase from the thermophilic Bacillus thermoleovorans strain A2: nucleotide sequence and analysis of the genes. FEMS Microbiol. Lett. 161:37-45.

9. Entsch, B., D. Ballou, and V. Massey. 1976. Flavin-oxygen derivatives involved in hydroxylation by $p$-hydroxybenzoate hydroxylase. J. Biol. Chem. 251:2550-2563.

10. Fieschi, F., V. Niviere, C. Frier, J.-L. Decout, and M. Fontecave. 1995. The mechanism and substrate specificity of the NADPH:flavin oxidoreductase from Escherichia coli. J. Biol. Chem. 270:30392-30400.

11. Firestone, D. 1978. The 2,3,7,8-tetrachlorodibenzo-para-dioxin problem: a review. Ecol. Bull. 27:39-52.

12. Fontecave, M., R. Eliasson, and P. Reichard. 1987. NAD(P)H:flavin oxidoreductase of Escherichia coli. A ferric iron reductase participating in the generation of the free radical of ribonucleotide reductase. J. Biol. Chem. 262:12325-12331.

13. Galan, N., E. Diaz, M. Prieto, and J. Garcia. 2000. Functional analysis of the small component of the 4-hydroxyphenylacetate 3-monooxygenase of Escherichia coli $\mathrm{W}$ : a prototype of a new flavin:NAD $(\mathrm{P}) \mathrm{H}$ reductase subfamily. $\mathrm{J}$ Bacteriol. 182:627-636.

14. Gibello, A., M. Suarez, J. L. Allende, and M. Martin. 1997. Molecular cloning and analysis of the genes encoding the 4-hydroxyphenylacetate hydrolase from Klebsiella pneumoniae. Arch. Microbiol. 267:160-166.

15. Haigler, B. E., W. C. Suen, and J. C. Spain. 1996. Purification and sequence analysis of 4-methyl-5-nitrocatechol oxygenase from Burkholderia sp. strain DNT. J. Bacteriol. 178:6019-6024.

16. Hastings, J., and C. Balny. 1975. The oxygenated bacterial luciferase-flavin intermediate. Reaction products via the light and dark pathways. J. Biol. Chem. 250:7288-7293.

17. Hubner, A., C. Danganan, L. Xun, A. Chakrabarty, and W. Hendrickson. 1998. Genes for 2,4,5-trichlorophenoxyacetic acid metabolism in Burkholderia cepacia AC1100: characterization of the $t f t C$ and $t f t D$ genes and locations of the $t f t$ operons on multiple replicons. Appl. Environ. Microbiol. 64:20862093.

18. Husain, M., B. Entsch, D. P. Ballou, V. Massey, and P. J. Chapman. 1980. Fluoride elimination from substrates in hydroxylation reaction catalyzed by p-hydroxybenzoate hydroxylase. J. Biol. Chem. 255:4189-4197.

19. Izumoto, Y., T. Mori, and K. Yamamoto. 1994. Cloning and nucleotide 
sequence of the gene for NADH:FMN oxidoreductase from Vibrio harveyi Biochim. Biophys. Acta 1185:243-246.

20. Kadiyala, V., and J. Spain. 1998. A two-component monooxygenase catalyzes both the hydroxylation of $p$-nitrophenol and the oxidative release of nitrite from 4-nitrocatechol in Bacillus sphaericus JS905. Appl. Environ. Microbiol. 64:2479-2484.

21. Kellogg, S., D. Chatterjee, and A. Chakrabarty. 1981. Plasmid-assisted molecular breeding: new technique for enhanced biodegradation of persistent toxic chemicals. Science 214:1133-1135.

22. Kilbane, J., K. Chatterjee, J. Karns, S. Kellogg, and A. Chakrabarty. 1982. Biodegradation of 2,4,5-trichlorophenoxyacetic acid by a pure culture of Pseudomonas cepacia. Appl. Environ. Microbiol. 44:72-78.

23. Koike, H., H. Sasaki, T. Kobori, S. Zenno, K. Saigo, M. E. Murphy, E. T. Adman, and M. Tanokura. 1998. 1.8 ̊ crystal structure of the major NAD(P)H:FMN oxidoreductase of a bioluminescent bacterium, Vibrio fischeri overall structure, cofactor and substrate-analog binding, and comparison with related flavoproteins. J. Mol. Biol. 280:259-273.

24. Laemmli, U. K. 1970. Cleavage of structural proteins during the assembly of the head of bacteriophage T4. Nature 227:680-685.

25. Lee, J.-Y., and L. Xun. 1998. Novel biological process for L-dopa production from L-tyrosine by $p$-hydroxyphenylacetate 3-hydroxylase. Biotechnol. Lett. 20:479-482.

26. Lei, B., M. Liu, S. Huang, and S. C. Tu. 1994. Vibrio harveyi NADPH-flavin oxidoreductase: cloning, sequencing, and overexpression of the gene and purification and characterization of the cloned enzyme. J. Bacteriol. 176: 3552-3558.

27. Lindstrom, K., J. Nordin, and F. Osterberg. 1981. Chlorinated organics of low and high relative molecular mass in pulp mill bleachery effluents, $p$ 1039-1058. In L. H. Keith (ed.), Advances in the identification and analysis of organic pollutants in water, vol. 2. Ann Arbor Science Publishers Inc., Ann Arbor, Mich.

28. Louie, T. M., C. M. Webster, and L. Xun. 2002. Genetic and biochemica characterization of a 2,4,6-trichlorophenol degradation pathway in Ralstonia eutropha JMP134. J. Bacteriol. 184:3492-3500.

29. Louie, T. M., H. Yang, P. Karnchanaphanurach, X. S. Xie, and L. Xun. 2002 FAD is a preferred substrate and an inhibitor of Escherichia coli general NAD(P)H:flavin oxidoreductase. J. Biol. Chem. 277:39450-39455.

30. Martin-Le Garrec, G., I. Artaud, and C. Capeillere-Blandin. 2001. Purification and catalytic properties of the chlorophenol 4-monooxygenase from Burkholderia cepacia strain AC1100. Biochim. Biophys. Acta 1547:288-301.

31. Massey, V. 1994. Activation of molecular oxygen by flavins and flavoproteins J. Biol. Chem. 269:22459-22462.

32. Miller, D. M., J. S. Olson, J. W. Pflugrath, and F. A. Quiocho. 1983. Rates of ligand binding to periplasmic proteins involved in bacterial transport and chemotaxis. J. Biol. Chem. 258:13665-13672.

33. Parry, R., and W. Li. 1997. An NADPH:FAD oxidoreductase from the valanimycin producer, Streptomyces viridifaciens: cloning, analysis, and overexpression. J. Biol. Chem. 272:23303-23311.

34. Prieto, M., and J. Garcia. 1994. Molecular characterization of 4-hydroxyphenylacetate 3-hydrolase of Escherichia coli. J. Biol. Chem. 269:2282322829 .

35. Robinson, J., and J. M. Cooper. 1970. Method of determining oxygen concentrations in biological media, suitable for calibration of oxygen electrode. Anal. Biochem. 33:390-399.

36. Sambrook, J., E. F. Fritsch, and T. Maniatis. 1989. Molecular cloning: a laboratory manual, 2nd ed. Cold Spring Harbor Laboratory Press, Cold Spring Harbor, N.Y.

37. Stintzi, A., Z. Johnson, M. Stonehouse, U. Ochsner, J. M. Meyer, M. L. Vasil, and K. Poole. 1999. The pvc gene cluster of Pseudomonas aeruginosa: role in synthesis of the pyoverdine chromophore and regulation by PtxR and PvdS. J. Bacteriol. 181:4118-4124.

38. Takizawa, N., H. Yokoyama, K. Yanagihara, T. Hatta, and H. Kiyohara. 1995. A locus of Pseudomonas pickettii DTP0602, had, that encodes 2,4,6trichlorophenol-4-dechlorinase with hydroxylase activity, and hydroxylation of various chlorophenols by the enzyme. J. Ferment. Bioeng. 80:318-326.

39. Tanner, J. J., B. Lei, S. C. Tu, and K. L. Krause. 1996. Flavin reductase P: structure of a dimeric enzyme that reduces flavin. Biochemistry 35:1353113539.

40. Velasco, A., S. Alonso, J. L. Garcia, J. Perera, and E. Diaz. 1998. Genetic and functional analysis of the styrene catabolic cluster of Pseudomonas sp. strain Y2. J. Bacteriol. 180:1063-1071.

41. Xun, L. 1996. Purification and characterization of chlorophenol 4-monooxygenase from Burkholderia cepacia AC1100. J. Bacteriol. 178:2645-2649.

42. Xun, L., and E. Sandvik. 2000. Characterization of 4-hydroxyphenylacetate 3-hydroxylase (HpaB) of Escherichia coli as a reduced flavin adenine dinucleotide-utilizing monooxygenase. Appl. Environ. Microbiol. 66:481-486.

43. Xun, L., and K. Wagnon. 1995. Purification and properties of component B of 2,4,5-trichlorophenoxyacetate oxygenase from Pseudomonas cepacia AC1100. Appl. Environ. Microbiol. 61:3499-3502.

44. Zaborina, O., D. Daubaras, A. Zago, L. Xun, K. Saido, T. Klem, D. Nikolic, and A. Chakrabarty. 1998. Novel pathway for conversion of chlorohydroxyquinol to maleylacetate in Burkholderia cepacia AC1100. J. Bacteriol. 180:4667-4675.

45. Zenno, S., K. Saigo, H. Kanoh, and S. Inouye. 1994. Identification of the gene encoding the major $\mathrm{NAD}(\mathrm{P}) \mathrm{H}$-flavin oxidoreductase of the bioluminescent bacterium Vibrio fischeri ATCC 7744. J. Bacteriol. 176:3536-3543. 